Influence of Ink Formulation and Drying Conditions on lonomer Distribution in High-Performance Roll-to-Roll-Coated Gas-Diffusion Electrodes

Scott Mauger (scott.mauger@nrel.gov) PRiME/236 th ECS Meeting, 101B-2218

October 6, 2020 


\section{Team}

NREL - Min Wang, K.C. Neyerlin, Tim van Cleve, Mike Ulsh

Colorado School of Mines - Michael Dzara, Svitlana Pylypenko Argonne National Lab - Firat Cetinbas, Jaehyung Park, Rajesh Ahluwalia, Debbie Myers

Carnegie Mellon University - Leiming Hu, Shawn Litster

Funding - D.O.E. Advanced Manufacturing Office, Roll-to-Roll Advanced Materials Manufacturing Lab Collaboration 


\section{Roll-to-Roll Manufacturing}

\section{REVIEW}

Electrocatalyst approaches and challenges for automotive fuel cells

Mark K. Debe ${ }^{1}$

$10 \%$ of 2030 market $=15 \mathrm{M}$ vehicles $/$ year

$=4.5 \mathrm{~B} \mathrm{MEAs} / \mathrm{year}$

$>20$ production lines - $585 \mathrm{MEAs} / \mathrm{min}$

$>$ Coating $-1 \mathrm{~m}$ wide $\times 20 \mathrm{~m} / \mathrm{min}$

High-performance R2R-manufactrured MEAs

needed to meet cost and volume targets 


\section{Direct Coating vs Decal Transfer}
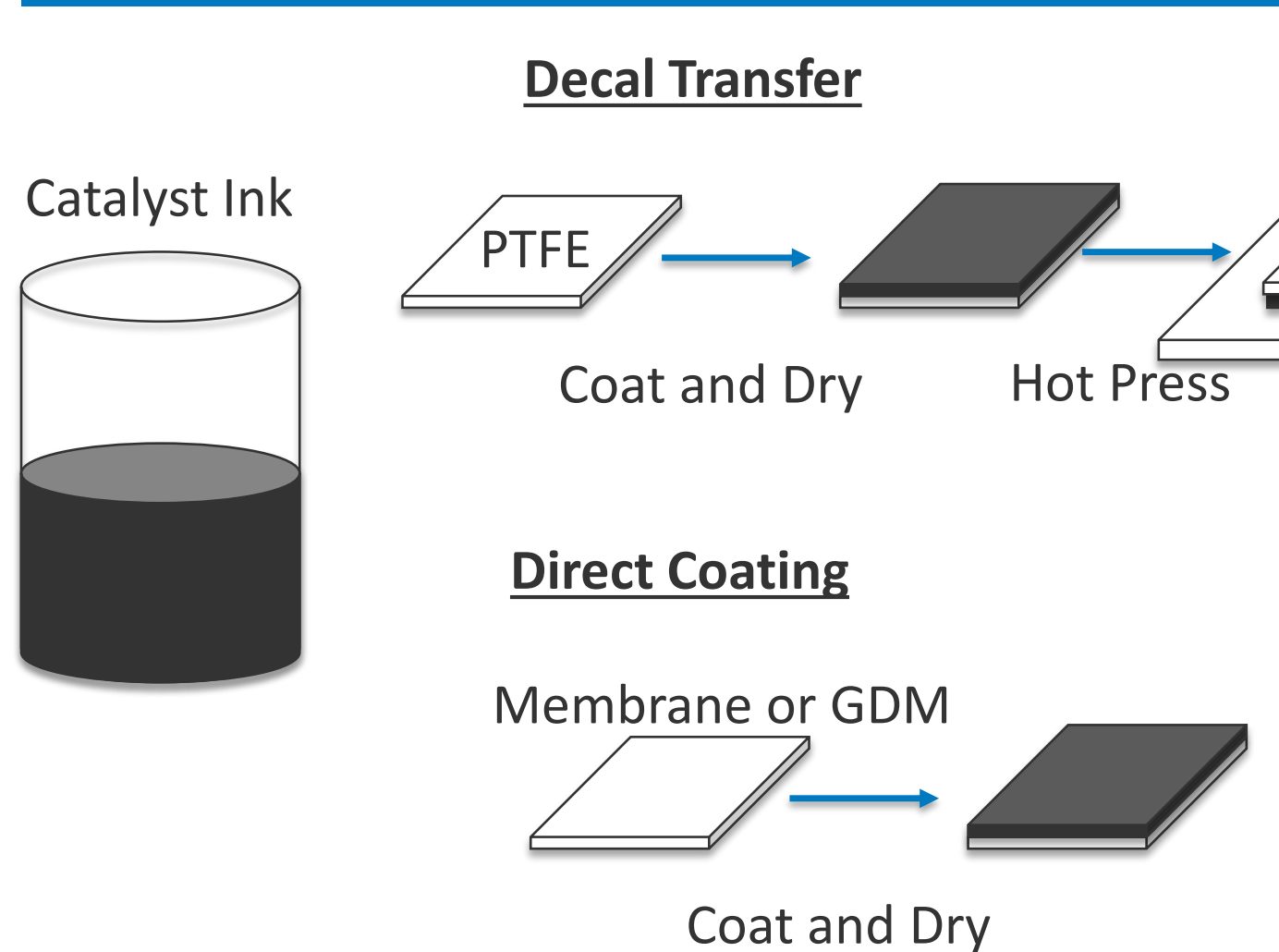

Coat and Dry 


\section{Motivation for Direct Coating}

\begin{tabular}{|l|c|c|c|c|c|c|}
\hline $\begin{array}{l}\text { Production Volume } \\
\text { (sys/yr) }\end{array}$ & 1000 & 10,000 & 20,000 & 50,000 & 100,000 & 500,000 \\
\hline $\mathrm{m}^{2}$ active area/yr & 7,470 & 74,702 & 149,404 & 373,511 & 747,022 & $3,735,111$ \\
\hline $\begin{array}{l}\text { Slot die coating process } \\
\left(\$ / \mathrm{m}^{2}\right)\end{array}$ & $\$ 52.59$ & $\$ 9.14$ & $\$ 4.92$ & $\$ 4.00$ & $\$ 2.93$ & $\$ 1.30$ \\
\hline
\end{tabular}

- Direct coating offers significant savings over decal coating

- Gas diffusion media easier to coat on than membrane 


\section{The GDE Challenge}

- Spray-coated GDEs often don't perform as well as CCMs

- Need an ionomer overlayer to form good GDE-membrane interface

Ionomer Overlayer

Cathode Layer: Catalyst \& lonomer

Microporous Layer

Gas Diffusion Layer

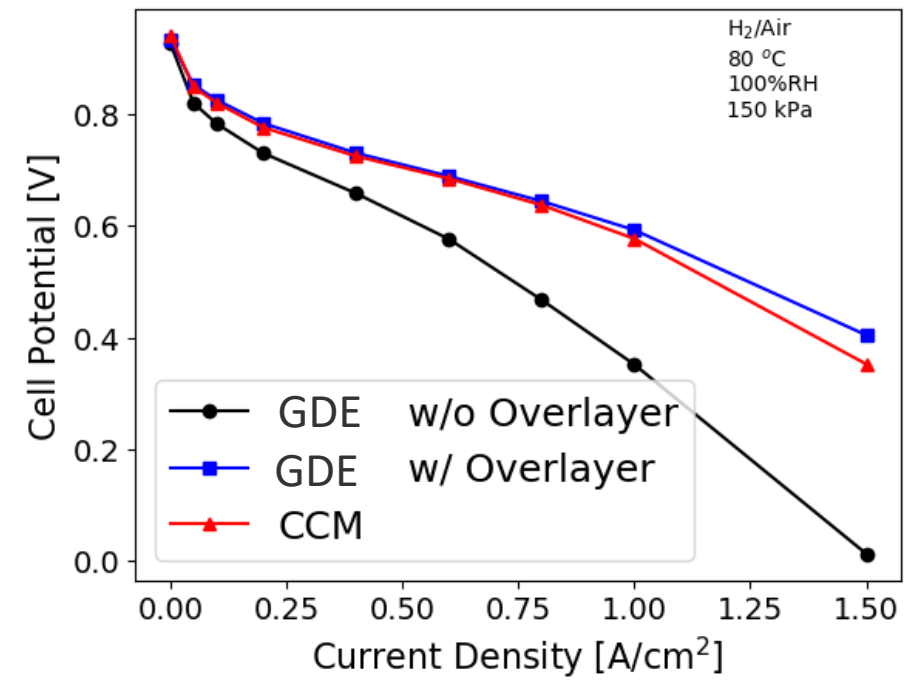

Two coating steps $(C L+$ overlayer) does not have an advantage over decal process 


\section{The Roll-to-Roll Advantage}

- In colloidal mixtures materials can phase separate form enriched surfaces

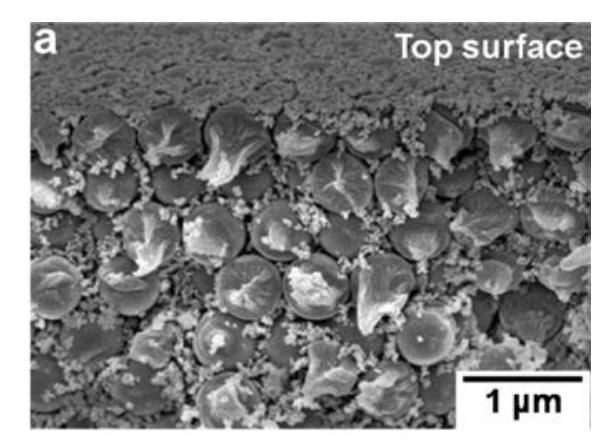

- Separation is favorable when:
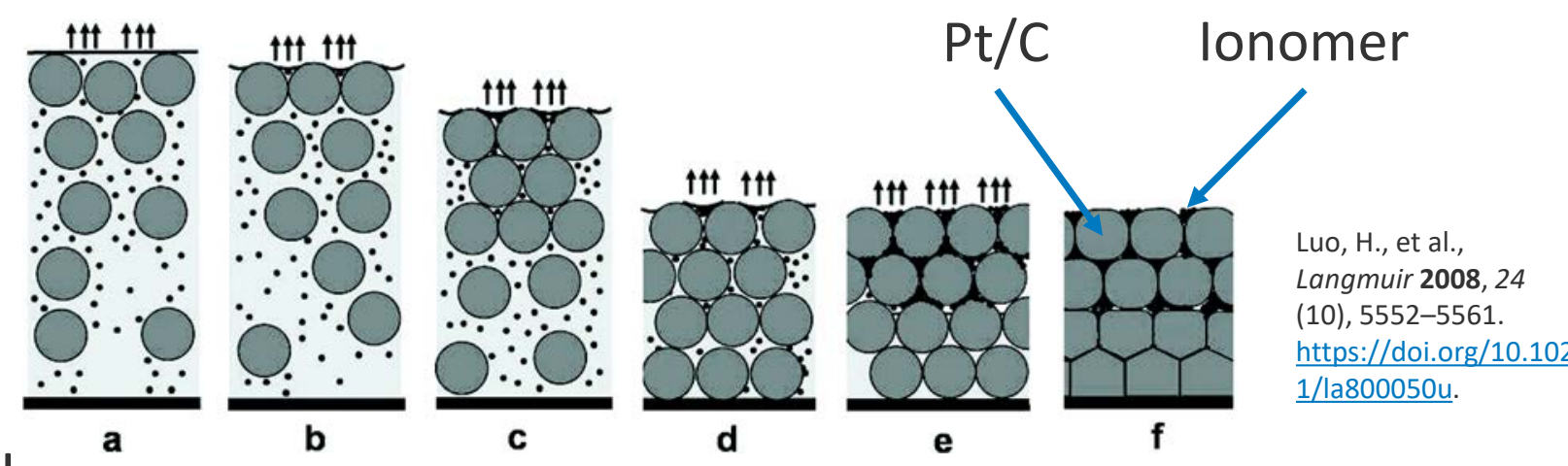

- Evaporation >> Diffusion, Sedimentation

- Large difference in particle size

- Higher concentration of small particles

- Goal-can we design an ink and single coating step that leads a GDE with an ionomer rich surface

Wet Catalyst Ink

Drying

lonomer-rich surface

Catalyst Layer 


\section{Small Scale Coating Trials}

- Mayer Rod coating on SGL 29BC diffusion media to simulate R2R coating

- Increase drying temperature to increase evaporation rate

- 25, 60, and $80^{\circ} \mathrm{C}$

- Increase ionomer:carbon ratio to increase volume of ionomer available to move to surface

$-0.9,1.2,1.6 \mathrm{I} / \mathrm{C}$

- Ink

- Pt/HSC (TKK TEC10E50E): 3.2 wt\%

- Dispersion Media: water/1-propanol (75/25 w/w)

- Nafion, 1000 EW 


\section{Measurement of Surface Ionomer}

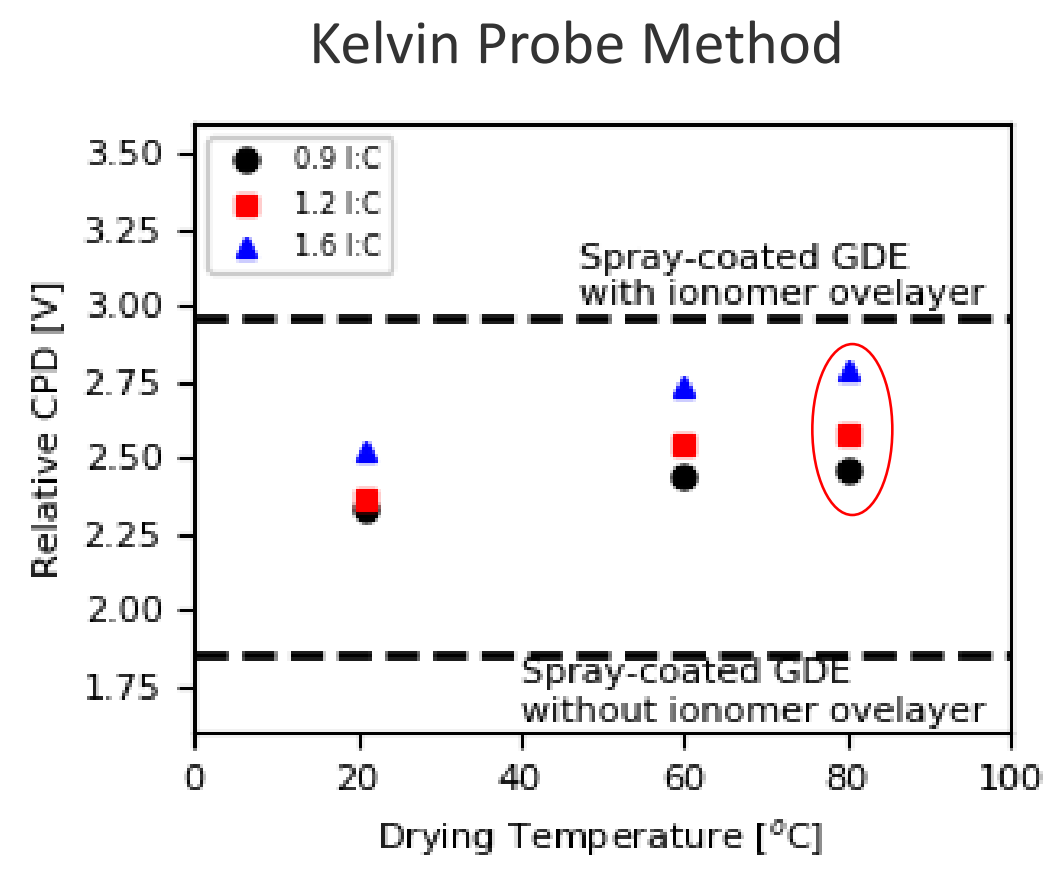

X-ray Photoelectron Spectroscopy

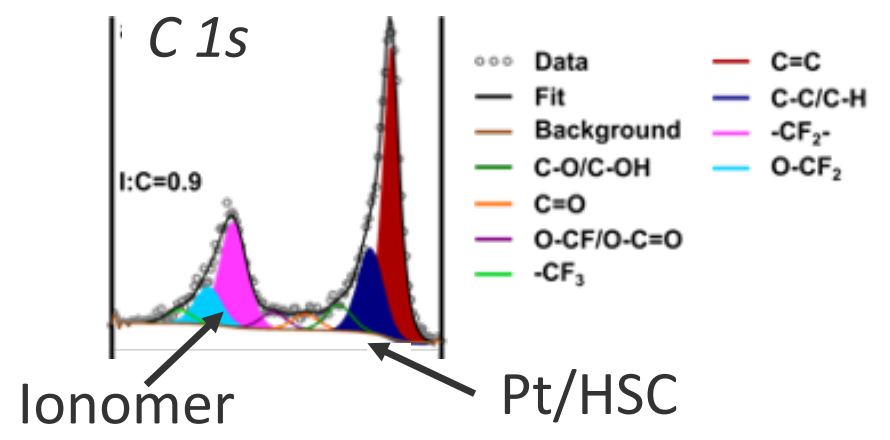

\begin{tabular}{|c|c|c|c|}
\hline Coating Method & I/C & $\mathbf{C}_{\mathbf{l}} / \mathbf{C}_{\mathbf{C}}$ & $\begin{array}{c}\Delta \mathbf{C P D} \\
{[\mathbf{m V}]}\end{array}$ \\
\hline $\begin{array}{c}\text { Spray-coated without overlayer } \\
\text { Spray-coated with overlayer }\end{array}$ & 0.9 & 0.21 & - \\
\hline Mayer Rod & 0.9 & 0.38 & 1104 \\
\hline & 1.2 & 0.49 & 605 \\
\hline & 1.6 & 0.58 & 726 \\
\hline
\end{tabular}

Increasing I/C and drying temp increase surface ionomer content 


\section{X-ray Computed Tomography}

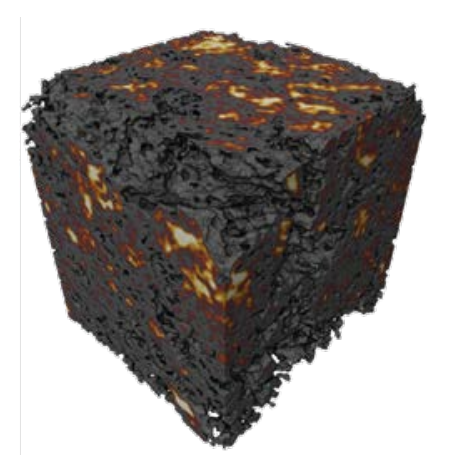

Increasing I/C:

- Increases conductivity

- Decrease diffusivity

\section{Local Ion Conductivity}
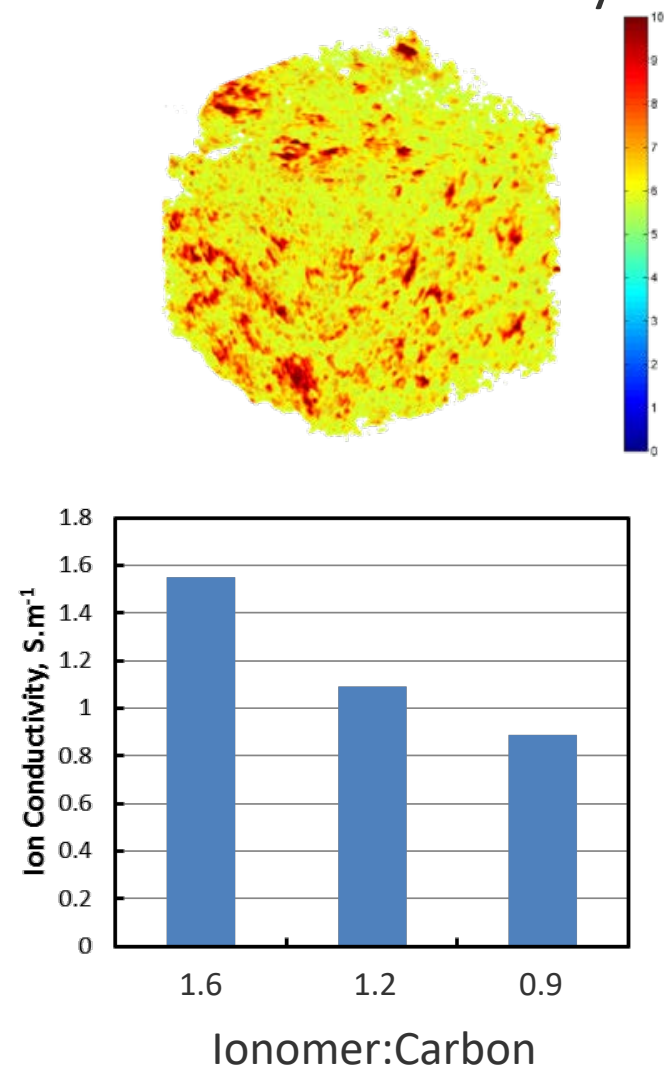

Local Diffusivity
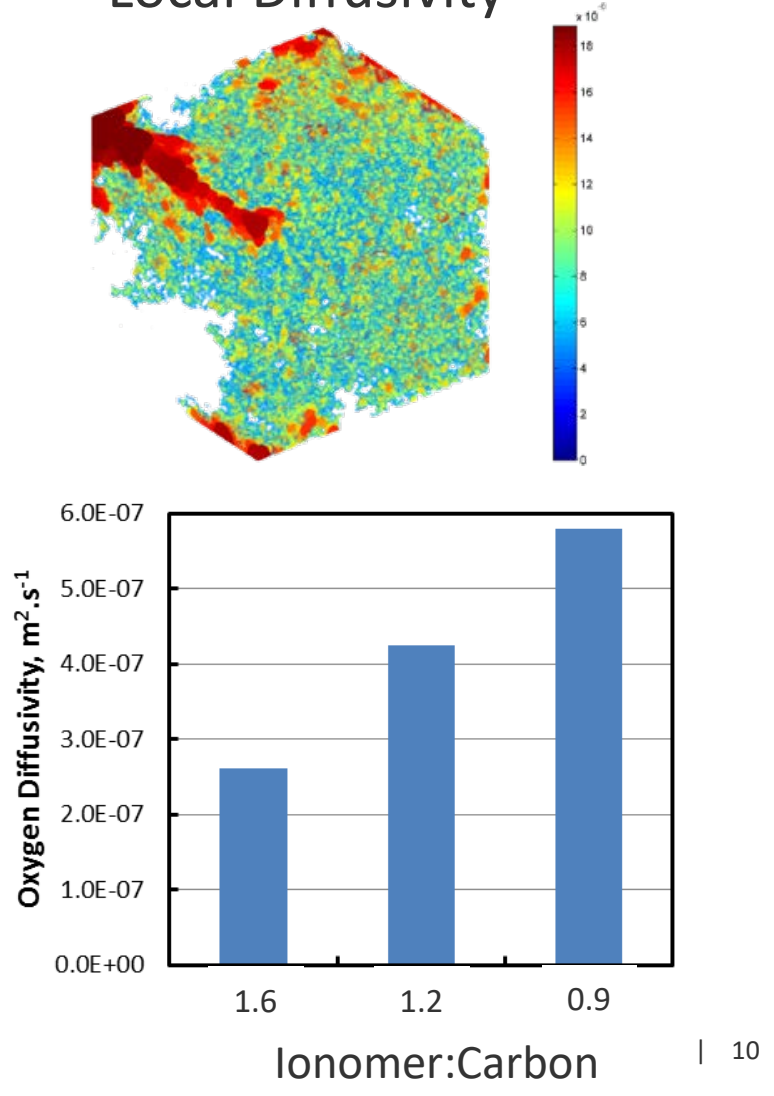


\section{Slot-Die-Coated GDEs}

- Same ink as rod coating

- Coating speed - $1 \mathrm{~m} / \mathrm{min}$

- Dry at $80{ }^{\circ} \mathrm{C}$

- Freudenberg H23C8 diffusion media

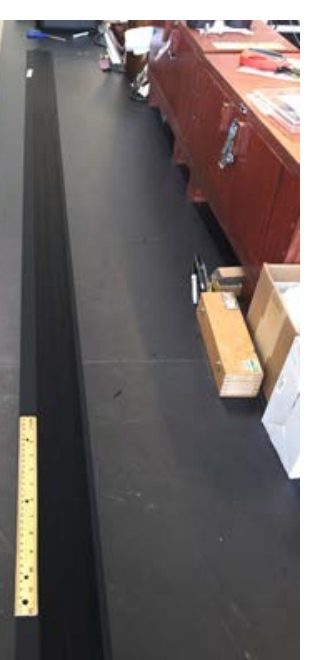

X-ray Photoelectron Spectroscopy

\begin{tabular}{l|l|l|}
\hline Coating Method & I/C & C $_{\mathbf{C}} \mathbf{C}_{\mathbf{S}}$ \\
\hline Spray - no overlayer & 0.9 & 0.21 \\
\hline Spray- overlayer & 0.9 & 0.38 \\
\hline & 0.9 & 0.63 \\
\hline R2R slot die & 1.2 & 1.4 \\
& 1.6 & 3.6 \\
\hline
\end{tabular}

R2R GDEs show same trends as rod coated 


\section{Fuel Cell Performance}
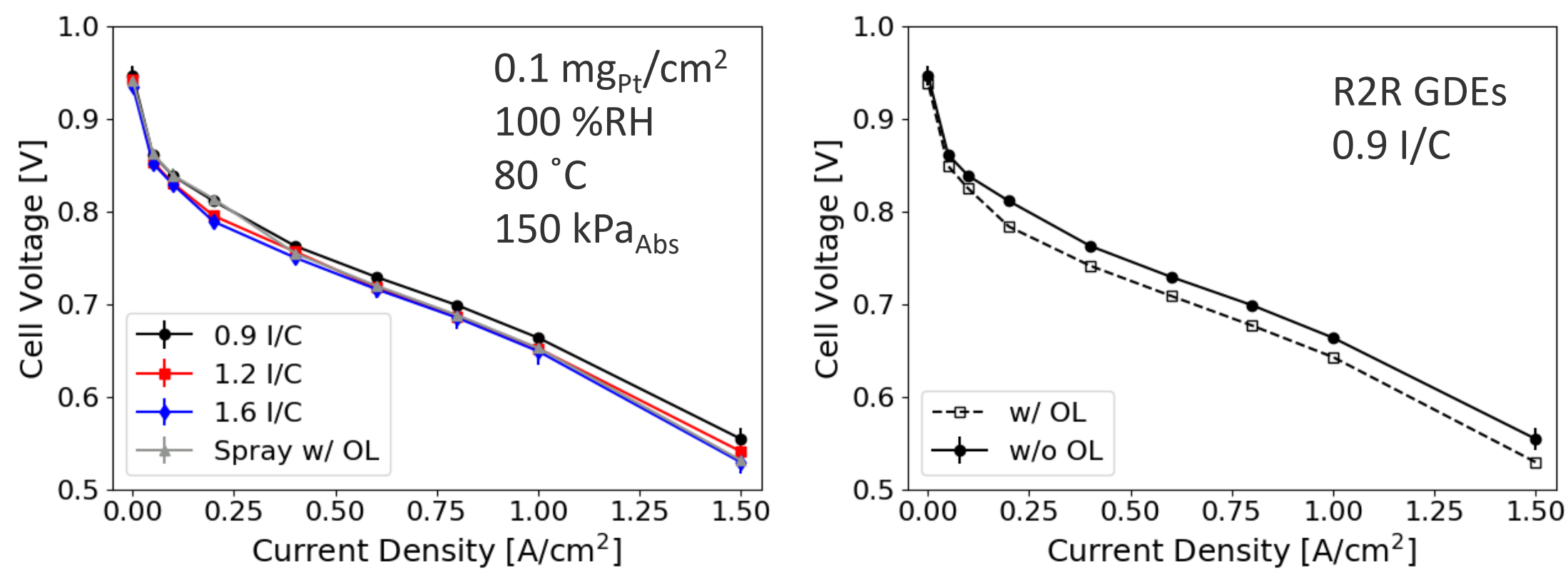

- 1-step R2R GDEs perform as well as 2-step spray-coated GDEs

- High performance also shown with PtCo-based R2R GDEs 


\section{$\mathrm{H}_{2} / \mathrm{N}_{2}$ Impedance Spectroscopy}

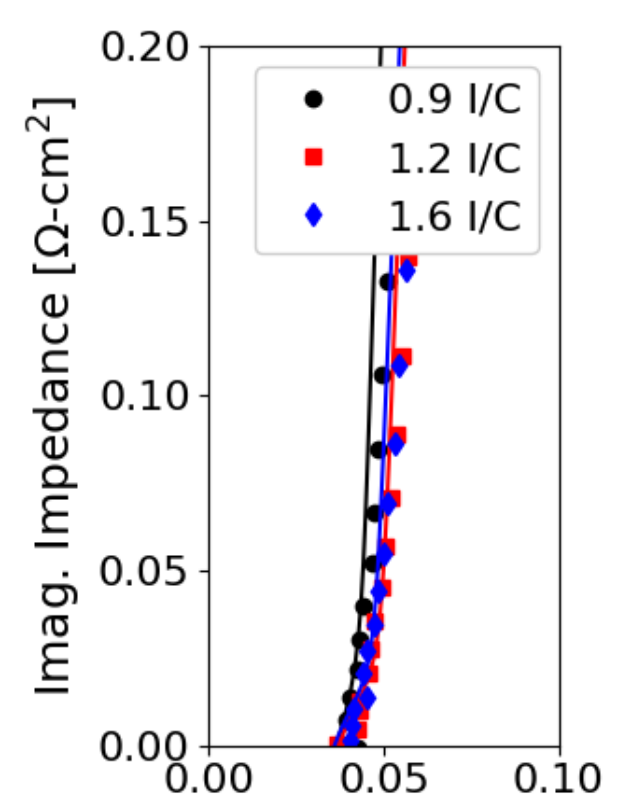

$$
Z(\omega)_{\text {model }}=j \omega L_{\text {wire }}+R_{\Omega}+\sqrt{\frac{R_{C L}}{Q_{D L}(j \omega)^{\phi}}} \operatorname{coth}\left(\sqrt{R_{C L} Q_{D L}(j \omega)^{\phi}}\right)
$$

\begin{tabular}{|c|c|c|}
\hline Coating Method & $\mathrm{I} / \mathrm{C}$ & $\begin{array}{c}\mathbf{R}_{\mathrm{CL}} \\
{\left[\mathbf{m} \Omega-\mathrm{cm}^{2}\right]}\end{array}$ \\
\hline Roll-to-Roll & 0.9 & $92 \pm 10$ \\
Slot Die & 1.2 & $88 \pm 10$ \\
\hline Spray - without ionomer overlayer & 1.6 & $81 \pm 10$ \\
\hline Spray - with ionomer overlayer & 0.9 & $222 \pm 10$ \\
\hline
\end{tabular}

Real Impedance $\left[\Omega-\mathrm{cm}^{2}\right]$

$0.9 \mathrm{l} / \mathrm{C}$ has sufficient ionomer to form good interface with membrane 


\section{Conclusions}

- Increasing drying temperature leads to ionomer enrichment at GDE surface

- Ionomer rich surface forms low resistance interface with membrane

- 1-coating step R2R GDEs have same performance as 2-step spray coated GDEs

- GDEs are viable for industrial manufacturing 


\title{
Scott Mauger
}

\section{scott.mauger@nrel.gov}

PRiME/236 th ECS Meeting

\section{I01B-2218}

\section{October 6, 2020}

\section{Thank You}

\section{www.nrel.gov}

NREL/PR-5900-76952

\begin{abstract}
This work was authored in part by the National Renewable Energy Laboratory, operated by Alliance for Sustainable Energy, LLC, for the U.S. Department of Energy (DOE) under Contract No. DE-AC36-08GO28308. The submitted manuscript was created, in part, by U Chicago Argonne, LLC, Operator of Argonne National Laboratory, Argonne, U.S. Department of Energy Office of Science laboratory, operated under Contract No. DE-AC02-06CH11357. This research used the resources of the Advanced Photon Source (APS), a U.S. Department of Energy (DOE) Office of Science User Facility, operated for the DOE Office of Science by Argonne National Laboratory, also under Contract No. DE-AC02 06CH11357. Funding provided by U.S. Department of Energy, Office of Energy Efficiency and Renewable Energy, Advanced Manufacturing Office. This work was conducted as part of the Roll-to-Roll Advanced Materials Manufacturing Laboratory Collaboration, and strongly leveraged work supported by the Hydrogen and Fuel Cell Technologies Office. The views expressed in the article do not necessarily represent the views of the DOE or the U.S. Government. The U.S. Government retains and the publisher, by accepting the article for publication, acknowledges that the U.S. Government retains a nonexclusive, paid-up, irrevocable, worldwide license to publish or reproduce the published form of this work, or allow others to do so, for U.S. Government purposes.
\end{abstract}

\section{GNREL}

Transforming ENERGY 